\title{
A new solid-phase extraction disk based on a sheet of single-walled carbon nanotubes
}

\author{
Hong Yun Niu • Ya Qi Cai • Ya Li Shi • Fu Sheng Wei • \\ Jie Min Liu • Gui Bin Jiang
}

Received: 11 June 2008 / Revised: 11 July 2008 / Accepted: 1 August 2008 / Published online: 24 August 2008

(C) Springer-Verlag 2008

\begin{abstract}
A new kind of solid-phase extraction disk based on a sheet of single-walled carbon nanotubes (SWCNTs) is developed in this study. The properties of such disks are tested, and different disks showed satisfactory reproducibility. One liter of aqueous solution can pass through the disk within 10-100 min while still allowing good recoveries. Two disks (DD-disk) can be stacked to enrich phthalate esters, bisphenol A (BPA), 4-n-nonylphenol (4-NP), 4-tert-octylphenol (4-OP) and chlorophenols from various volumes of solution. The results show that SWCNT disks have high extraction ability for all analytes. The SWCNT disk can extract polar chlorophenols more efficiently than a $\mathrm{C}_{18}$ disk from water solution. Unlike the activated carbon disk, analytes adsorbed by the new disks can be eluted completely with $8-15 \mathrm{~mL}$ of methanol or acetonitrile. Finally, the DD-disk system is used
\end{abstract}

Electronic supplementary material The online version of this article (doi:10.1007/s00216-008-2332-1) contains supplementary material, which is available to authorized users.

H. Y. Niu • Y. Q. Cai · Y. L. Shi · G. B. Jiang

State Key Laboratory of Environmental Chemistry and

Ecotoxicology, Research Center for Eco-Environmental Science,

Chinese Academy of Sciences,

Beijing 100085, China

\section{F. S. Wei $(\bowtie)$}

China National Environmental Monitoring Center,

Beijing 100029, China

e-mail: weifs@public3.bta.net.cn

\section{J. M. Liu}

Department of Chemistry,

University of Science and Technology Beijing,

Beijing 100083, China

\section{Y. Q. Cai $(\square)$}

Chinese Academy of Sciences,

Research Center for Eco-Environmental Sciences,

P.O. Box 2871, Beijing 100085, China

e-mail: caiyaqi@rcees.ac.cn to pretreat $1000-\mathrm{mL}$ real-world water samples spiked with BPA, 4-OP and 4-NP. Detection limits of 7, 25, and $38 \mathrm{ng} \mathrm{L}^{-1}$ for BPA, 4-OP, and 4-NP, respectively, were achieved under optimized conditions. The advantages of this new disk include its strong adsorption ability, its high flow rate and its easy preparation.

Keywords Solid-phase extraction disk · Single-walled carbon nanotube sheet $\cdot \mathrm{C}_{18}$ disk . Activated carbon disk . Large-volume water sample

\section{Introduction}

The solid-phase extraction (SPE) disk introduced by $3 \mathrm{M}$, Inc. in 1989 as an advanced technique for sample preparation is more suitable than a SPE cartridge for treating largevolume environmental samples [1-3]. Disks have some distinct advantages over conventional SPE cartridges: they enable faster flow rates and less channeling, and they reduce the risk of plugging and pollution [1-4]. Various adsorbents are available, such as $\mathrm{C}_{18}$ [2-5], styrenedivinylbenzene copolymer $[2,4]$, activated carbon and graphitized carbon black (GCB) [6], and so forth. It is well known that $\mathrm{C}_{18}$ adsorbent shows insufficient extraction efficiency towards polar analytes. The advantage of using activated carbon and GCB adsorbents in SPE methods is their high adsorption abilities with respect to nonpolar and polar analytes; but they also show irreversible adsorption and low recoveries for some analytes [7]. Therefore, a new kind of SPE disk is required that combines the merits of $\mathrm{C}_{18}$ and $\mathrm{GCB} /$ activated carbon adsorbents.

Recently, carbon nanotubes (CNTs), including singlewalled carbon nanotubes (SWCNTs) and multiwalled carbon nanotubes (MWCNTs), have shown high potential when applied to trap nonpolar [8-10], moderately polar [11, 12] and highly polar analytes [13] in aqueous solutions. 
Moreover, the adsorbed analytes can be desorbed completely with a water-miscible solvent such as methanol or acetonitrile. They have therefore been regarded as promising SPE adsorbents for pretreating environmental water samples. However, in most of these works, the CNT adsorbents are used in the form of cartridges; thus far, there have been few reports on their application as SPE disks.

There are several reports on the use of CNT sheets in the fields of electronics and material science [14-24]. A CNT sheet, also called "bucky paper," is typically prepared by the vacuum filtration of a certain volume of CNT suspension through a filter [14, 22]. The sheet produced is allowed to dry and then peeled from the filter. CNT sheets can be readily assembled from SWCNTs, but are much more difficult to produce consistently from the more rigid MWCNTs [22]. SWCNT sheets have shown suitable mechanical properties and have been used as alternative actuators [14-17], electrolytes [18, 19], artificial muscles [20], and sensors [21]. The SWCNT sheet possesses a much larger specific surface area than the SWCNT particles, and a sheet (47 mm in diameter) made from $\sim 30 \mathrm{mg}$ of SWCNTs is typically $60 \mu \mathrm{m}$ in thickness with a specific surface area of $\sim 700 \mathrm{~m}^{2} / \mathrm{g}$ [22]. Zheng [22] has packed a SWCNT sheet into a metal tube to sample organic vapor, and the good results obtained reflect its potential for use in ambient air monitoring for environmental and security applications. Based on the special properties of SWCNT sheets and the abovementioned facts, we supposed that it should be feasible to use a SWCNT sheet as a SPE disk to extract organic compounds from large-volume environmental water samples.

In this paper, the SWCNT disks were prepared with a qualitative filter paper as support, and they were not peeled from the filter in order to improve their mechanical properties. The purpose of this work was mainly to demonstrate the validity of the SWCNT sheet as a SPE disk. A comparison between a commercial $\mathrm{C}_{18}$ disk and an activated carbon disk was performed. Four phthalate esters, BPA, 4-NP and 4-OP, and five chlorophenols were selected for study. Finally, the SWCNT disks were used to extract BPA, 4-OP, and 4-NP from several natural water samples.

\section{Materials and methods}

\section{Reagents}

The model compounds included 4-n-nonylphenol, 4-tertoctylphenol, bisphenol A; di- $n$-propylphthalate, di- $n$-butylphthalate, dicyclohexylphthalate and di- $n$-octylphthalate;
Fig. 1 TEM images of SWCNTs before (A) and after (B) purification; image of SWCNT disk (C) and SEM image of the SWCNT surface (D)
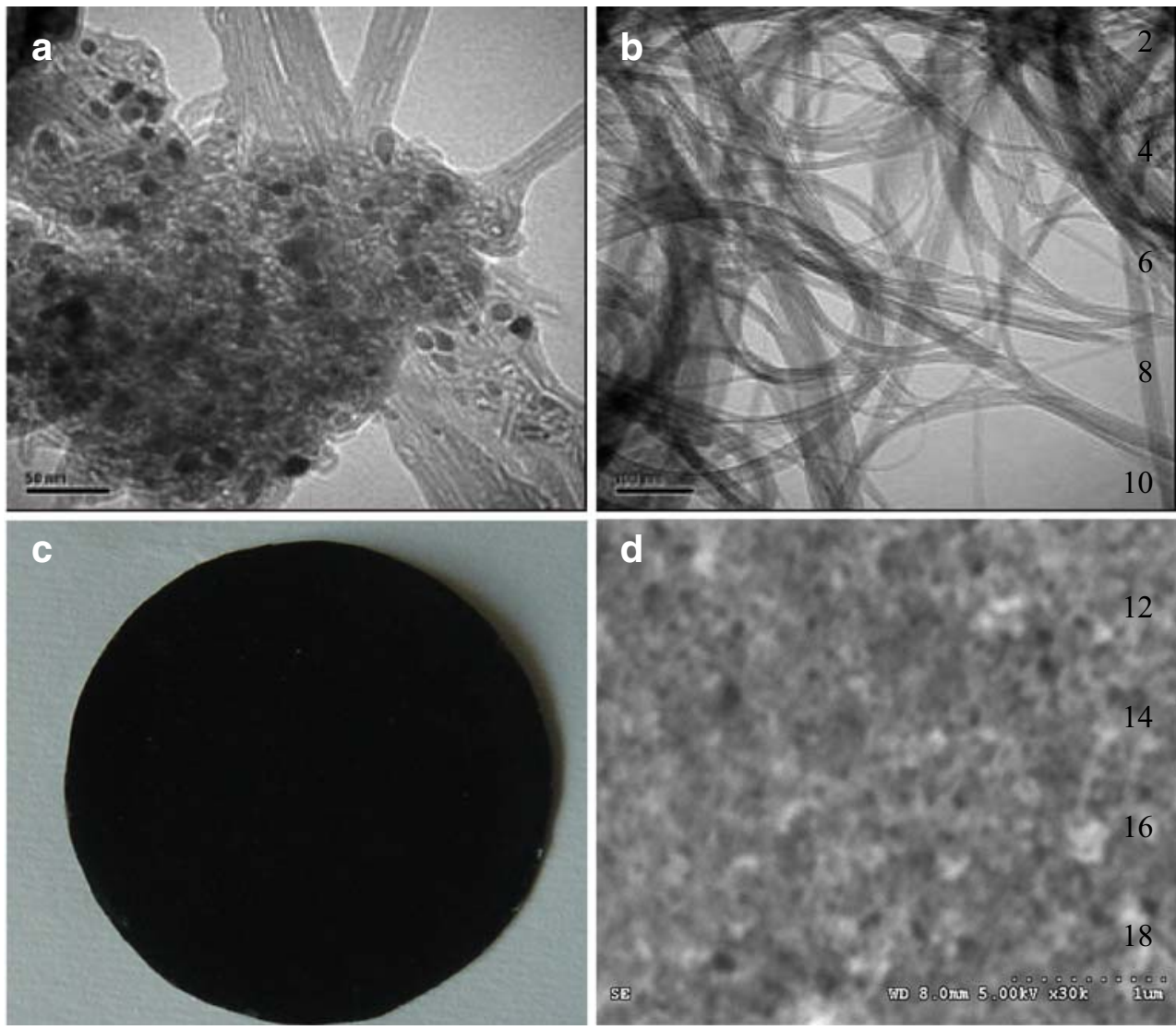
3-chlorophenol, 4-chlorophenol, 2,4-dichlorophenol, 2,4, 6-trichlorophenol and pentachlorophenol. All the analytes and organic solvents were highly purified (see the "Electronic Supplementary Material"). The SWCNTs were kindly donated by Nankai University (Tianjin, China). The purity of the as-received SWCNTs was about $50-80 \mathrm{wt} \%$, and the length an individual SWCNT was in the range of hundreds of nanometers to $20 \mu \mathrm{m}$. The average external and internal diameters of the SWCNTs were in the ranges $1.5-2.5 \mathrm{~nm}$ and $1.0-2 \mathrm{~nm}$, respectively, as observed using a transmission electron microscope (TEM; JEM-2011, JEOL, Tokyo, Japan) with an acceleration voltage of $200 \mathrm{kV}$. The specific surface area of the SWCNTs, as determined by a BET method, were $297.72 \mathrm{~m}^{2} \mathrm{~g}^{-1}$. Before preparing the SWCNT disk, the SWCNTs were purified. First, the asreceived SWCNTs were ultrasonicated in concentrated $\mathrm{HCl}$ for $2 \mathrm{~h}$. After filtration and washing with plenty of deionized water at $\mathrm{pH} 7.0$, the black solid was heated at $200{ }^{\circ} \mathrm{C}$ for $24 \mathrm{~h}$. The purity of the SWCNTs increased to $90-95 \%$ after treatment. TEM images of as-received and purified SWCNTs are shown in Fig. 1. Qualitative filter paper (HangZhou, China) used to support the SWCNTs; the sorbent was $0.18-0.20 \mathrm{~mm}$ in thickness and the diameter of the filter was about $47 \mathrm{~mm}$. The ENVI ${ }^{\mathrm{TM}}-18$ DSK SPE disk and the Empore ${ }^{\mathrm{TM}}$ activated carbon disk $(\Phi=47 \mathrm{~mm}, 500 \mathrm{mg})$ were purchased from Supelco (Bellefonte, PA, USA) and 3M, Inc. (St. Paul, MN, USA), respectively.

\section{Chromatographic conditions}

Analytes were detected using a Dionex SUMMIT ${ }^{\circledR}$ HPLC system (Dionex, Sunnyvale, CA, USA) with a Dionex RF 2000 fluorescence detector (FLD) for BPA, 4-OP and 4-NP detection, and a PDA-100 photodiode array detector for detecting other compounds. The separation conditions are described in the "Electronic Supplementary Material."

\section{Preparation of the SPE disk}

To produce a SPE disk, $30 \mathrm{mg}$ of SWCNTs was ultrasonicated in $50 \mathrm{~mL} 5 \%$ sodium dodecyl sulfate (SDS) solution for $30 \mathrm{~min}$. The suspension was filtered through a qualitative filter paper $47 \mathrm{~mm}$ in diameter using a vacuum pump. By doing so, the SWCNT sorbents were distributed
Fig. 2 Effect of the number of disks on the recoveries of BPA, 4-OP and 4-NP, obtained by extracting different volumes of standard solutions spiked with $100 \mathrm{ng} \mathrm{mL}^{-1}$ of each analyte. A Single disk; B double disk; C triple disk. Diamonds, bisphenol A; squares, 4-tertoctylphenol; triangles, 4- $n$ nonylphenol
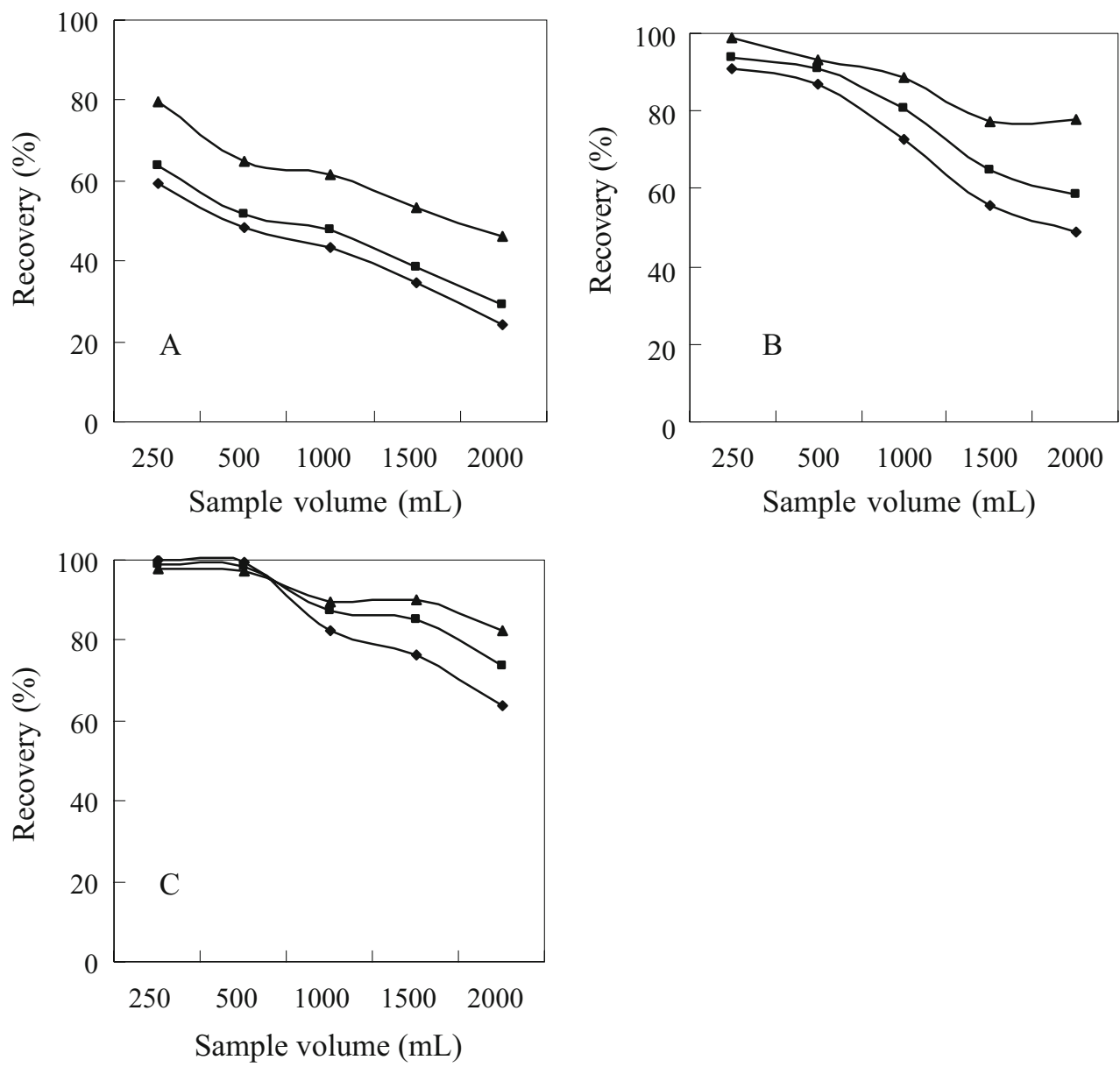
onto the filter uniformly. To get rid of the residual SDS, the disk was washed with methanol and water successively. It was finally dried at room temperature. Images of the SWCNT disk are shown in Fig. 1C and D.

Solid-phase extraction and sample loading

Water samples were extracted onto the SWCNT disks, the $\mathrm{C}_{18}$ disk or the activated carbon disk using a vacuum filtration apparatus. Prior to use, the disks were conditioned with $10 \mathrm{~mL}$ of methanol and then $10 \mathrm{~mL}$ of water. Different volumes of samples spiked with analytes were passed through the preconditioned disks. After sample extraction, the disks were allowed to dry in air under vacuum for $5 \mathrm{~min}$; thereafter, analytes were eluted into test tubes with eluent. In this step, the elution solvent was rinsed down the sides of the glass filtration apparatus. The eluent was concentrated on a rotary evaporator to $0.5 \mathrm{~mL}$ or dryness (for methylene chloride, acetone, and tetrahydrofuran solvent) and reconstituted in $1.0 \mathrm{~mL}$ of acetonitrile. Twenty-microliter aliquots were injected into the HPLC system for analysis.

A tap water sample was collected from our laboratory. A river water sample was collected from the Jing Mi canal river (Beijing), and a wastewater sample was taken from the effluent discharge gate of the biggest municipal wastewater treatment plant. The water samples were filtered through $0.22-\mu \mathrm{m}$ filters to remove suspended solids. One liter of the water sample or the water sample spiked with analytes was passed through the preconditioned disks for sample analysis or recovery tests.

\section{Results and discussion}

\section{SWCNT disks}

Due to the strong van der Waals interactions for radii of just a few nanometers, the SWCNTs exhibited strong adhesion to each other. The SWCNTs were arranged in bundles with close-packed stacking, forming self-organized cables [24]. The highly adhesive structure of the raw SWCNTs resulted in difficulties with gas adsorption accessibility [19]. Therefore, the measured surface area of the raw SWCNTs was significantly smaller than the theoretical BET area $\left(3000 \mathrm{~m}^{2} \mathrm{~g}^{-1}\right.$ for open nanotubes $3 \mathrm{~nm}$ in diameter with $0.4 \mathrm{~nm}$ spacing between the walls of two adjacent tubes) [25]. Most importantly, the adhesive structure of the raw SWCNTs led to an uneven distribution of particles on the filter surface [20]. A debundling process to open the ends of the tubes was therefore necessary prior to bucky paper production by filtration. Surfactant (or organic solvent [25]) treatment and ultrasonic processes [24] can aid the
Fig. 3 Effect of sample volume on the recoveries of phthalate ester analytes using the SWCNT DD-disk system $(\mathbf{A})$, an ENVI ${ }^{\mathrm{TM}}-18$ DSK disk (B) and an Empore ${ }^{\mathrm{TM}}$ activated carbon disk (C). Diamonds, di- $n$ propylphthalate; squares, di- $n$-butylphthalate; triangles, dicyclohexylphthalate; crosses, dioctylphthalate. Concentration of each analyte: $10 \mathrm{ng} \mathrm{mL}^{-1}$. Volume of eluent: DD-disk system, methanol, $15 \mathrm{~mL}$; ENVITM-18 DSK disk, methanol, $7 \mathrm{~m}$; Empore ${ }^{\mathrm{TM}}$ activated carbon disk, methylene chloride, $80 \mathrm{~mL}$
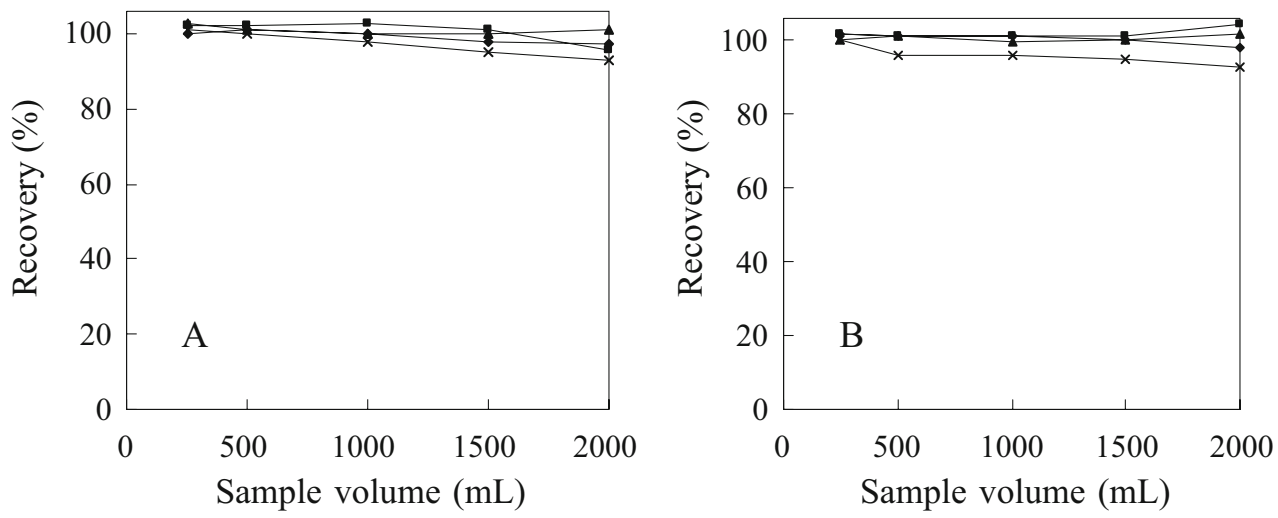
Fig. 4 Effect of the sample volume on the recoveries of BPA, 4-OP and 4-NP when using the SWCNT DD-disk system (A) or an ENVI ${ }^{\mathrm{TM}}-18$ DSK disk (B). Diamonds, bisphenol A; squares, 4-tertoctylphenol; triangles, 4- $n$ nonylphenol. Concentration of each analyte: $10 \mathrm{ng} \mathrm{mL}^{-1}$.

Volume of eluent (methanol): DD-disk system, $10 \mathrm{~mL}$; ENVI $^{\text {TM}}-18$ DSK disk, $7 \mathrm{~mL}$

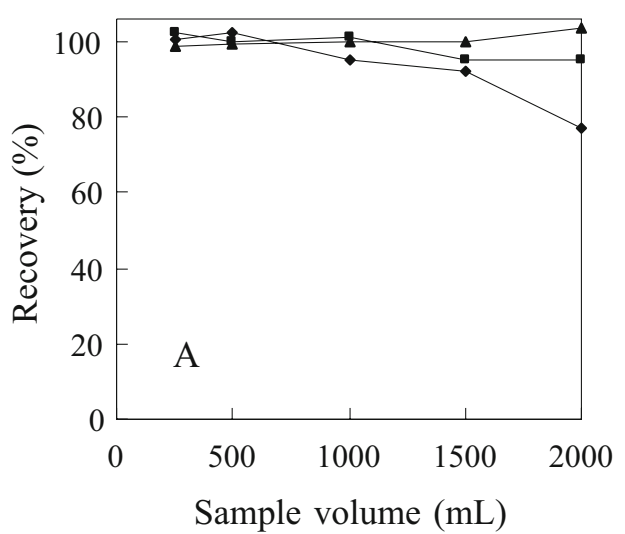

homogenization of the particles, reduce bundle size and enhance the external area of the SWCNTs, as shown by the experiment of Zheng et al. [22]. During the filtration process, the dispersed SWCNTs distributed homogeneously on the filter surface. Due to the strong van Waals forces among the tubes, the framework of the bucky paper also consists of SWCNT bundles [14]. However, the bundles were well ordered with small diameters. The interactions between the nanotubes were so strong that the stress generated by the nanotube sheets perpendicular to the disk surface was about five times higher than that of human skeletal muscle [16]. Therefore, these nanotubes can be fixed onto the filter surface without leaking or peeling during the processes of filtration or sample loading. In our study, the sheet was typically $60 \mu \mathrm{m}$ thick, as measured with a micrometer, and had density of $\sim 0.25 \mathrm{~g} \mathrm{~cm}^{-3}$, based on the mass, area, and thickness of the sheet.

The SWCNTs had to be purified to obtain good reproducibility of the SWCNT disk preparation process. The density of disks made from the as-received SWCNTs was about $0.16 \mathrm{~g} \mathrm{~cm}^{-3}$, which was clearly less than the density of those prepared with purified SWCNTs. A sufficient amount of SDS (no less than $2.5 \mathrm{wt} \%$ in solution) was also essential in order to disperse the SWCNTs thoroughly and thus make sure that all of the adsorbents distribute onto the supporting material uniformly. When the concentration of SDS was insufficient, an agglomerate of SWCNTs was observed in the suspension. Under these optimal conditions, disks of identical thickness could be prepared.

\section{Choice of the supporting material}

Different kinds of membranes or filters were tested for SWCNT disk preparation, including polycarbonate membrane, nylon filter, cellulose membrane and qualitative filter paper (QFP). Since the cellulose membrane was soluble in organic solvents, such materials were not suitable to act as supporting material. The surfaces of the polycarbonate membrane and the nylon filter were so smooth that the SWCNTs sheet was easily peeled from the membrane after the disk had dried, so these were not selected for disk preparation. The cheapest of the filters, QFP, had a rough surface that enabled the SWCNT sheet to maintain a tight grip on the filter. Moreover, this filter can endure most organic solvents (nonpolar, moderately polar or polar solvents), and so it would not impede any potential applications of the disk. Its perfect mechanical properties also protect the disk against fracture and crinkle.
Fig. 5 Effect of the sample volume on the recoveries of chlorophenol analytes using the SWCNT DD-disk system (A) or an ENVITM-18 DSK disk (B). Diamonds, 4-chlorophenol; squares, 3-chlorophenol; triangles, 2,4-dichlorophenol; crosses, 2,4,6-trichlorophenol; asterisks, pentachlorophenol. Concentration of each analyte: $10 \mathrm{ng} \mathrm{mL}^{-1}$. Volume of eluent (methanol): DD-disk system, $8 \mathrm{~mL}$; ENVI ${ }^{\mathrm{TM}}-18$ DSK disk, $7 \mathrm{~mL}$
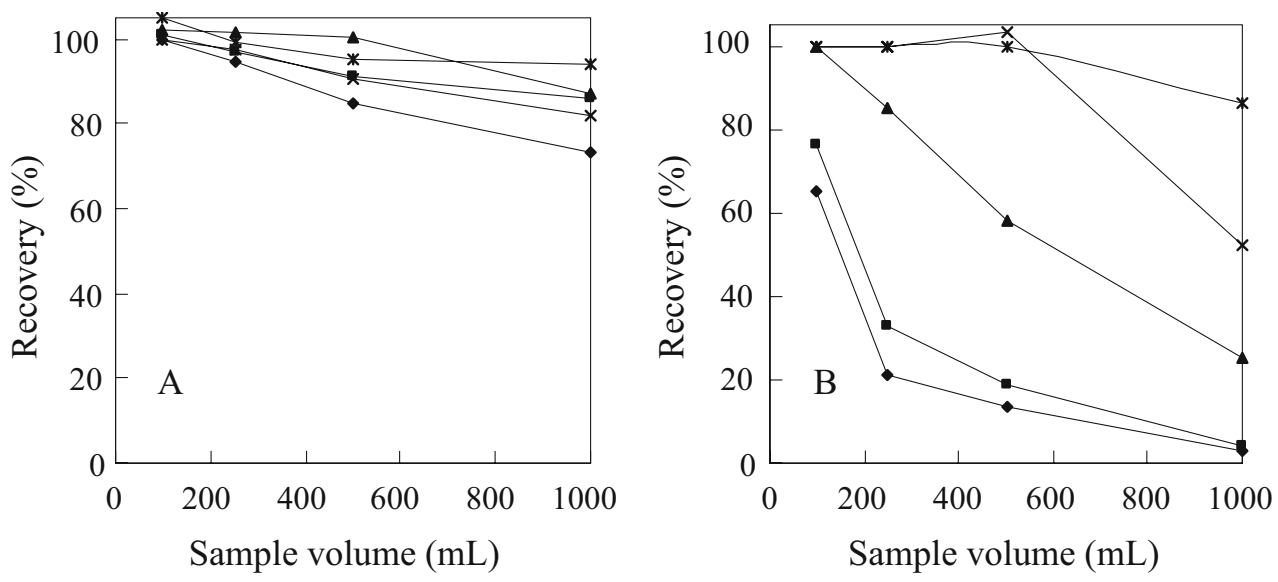
Table 1 Linear ranges, calibration equations, correlation coefficients and detection limits for the analytical system ${ }^{\mathrm{a}}$

\begin{tabular}{lllll}
\hline Compound & Linear range $\left(\mathrm{ng} \mathrm{mL}^{-1}\right)$ & Calibration equations & $R^{2}$ & Detection limit $\left(\mathrm{ng} \mathrm{L}^{-1}\right)$ \\
\hline Bisphenol A & $0.10-20.00$ & $y=0.3677 x-0.0169$ & 0.9991 & 7 \\
4-tert-Octylphenol & $0.10-20.00$ & $y=0.3408 x+0.0573$ & 0.9962 & 25 \\
4- $n$-Nonylphenol & $0.10-20.00$ & $y=0.3276 x+0.0532$ & 0.9986 & 38 \\
\hline
\end{tabular}

a The DD-disk system was used

\section{Reproducibility of the SWCNT disks}

To investigate this property of the SWCNT disks, three alkylphenols (BPA, 4-NP and 4-OP) were selected as model compounds. The reproducibility of the recoveries of the selected analytes extracted by the SWCNT disks, expressed as the relative standard deviations (RSDs; $n=5$ ) for $500 \mathrm{~mL}$ of purified water spiked at $100 \mathrm{ng} \mathrm{mL}^{-1}$ were lower than $5 \%$. The RSDs of seven disks tested were found to be less than $15 \%$.

\section{Effect of number of layers of stacked disks}

Because the disks are only $0.25 \mathrm{~mm}$ in thickness, they can easily be stacked for applications without leaking. To enhance the adsorption capacity of the disks, double or even triple disks were used together. The eluent volumes needed for different layered-disk systems were compared by treating $500 \mathrm{~mL}$ of purified water spiked with $10 \mathrm{ng} \mathrm{mL} \mathrm{m}^{-1}$ of alkylphenols. It was turned out that the required eluent volume did not change greatly as the number of layers was increased, and the volumes needed in this experiment were 7, 9, and $12 \mathrm{~mL}$ for the single-disk (SD), double-disk (DD), and triple-disk (TD) systems, respectively.

To investigate the effect of the numbers of layers on the recoveries of the analytes, different volumes of aqueous solution spiked with $100 \mathrm{ng} \mathrm{mL} \mathrm{m}^{-1}$ of alkylphenols were extracted by the SD, DD, or TD systems. The results are shown in Fig. 2. It can be seen that the recoveries of the analytes were low when they were extracted by the SD system, and the highest recoveries obtained were in the range of $60-80 \%$; moreover, the recoveries dropped significantly with increasing sample volume. These low recoveries possibly resulted from the incomplete sorption of the analytes onto the disks because of the considerable amounts present in each solution. However, it would be helpful to observe an increase in the recovery with an increasing number of disks. The recoveries were greatly enhanced when the DD and TD systems were used; the highest recoveries of three analytes increased to $100 \%$. The trapping efficiency of the different systems were compared by deriving the corresponding recovery ratios $(\mathrm{DD} / \mathrm{SD}$, or TD/DD) for the analytes. For BPA and 4-OP, the DD/SD ratio gradually rose from 1.5 to 2.0 , and the ratio for 4-NP changed from 1.2 to 1.7 , indicating that the trapping efficiency of the second disk increased with increasing sample volume. However, the TD/DD ratios for BPA and 4OP were in the range of 1.1-1.3; and for 4-NP, the corresponding ratios ranged from 1.0 to 1.1 . This meant that the third disk in the TD system was not being utilized completely. Therefore, the DD system improved the extraction ability of the SWCNT disks more efficiently than the TD system.

Table 2 Results from the determinations and recoveries of real-world water samples spiked with three target analytes ${ }^{\mathrm{a}}$

\begin{tabular}{|c|c|c|c|c|c|c|c|}
\hline \multirow[t]{2}{*}{ Water sample } & \multirow[t]{2}{*}{ Spiked $\left(\mathrm{ng} \mathrm{mL} \mathrm{m}^{-1}\right)$} & \multicolumn{3}{|c|}{$\begin{array}{l}\text { Detected } \\
\left(\mathrm{ng} \mathrm{mL}^{-1}\right)\end{array}$} & \multicolumn{3}{|c|}{ Recovery $(\%)^{b}$} \\
\hline & & BPA & 4-OP & 4-NP & BPA & 4-OP & 4-NP \\
\hline \multirow[t]{3}{*}{ Tap water } & 0.00 & $n d^{c}$ & nd & nd & & & \\
\hline & 0.50 & 0.48 & 0.50 & 0.47 & $94.9 \pm 6$ & $99.7 \pm 4$ & $94.8 \pm 4$ \\
\hline & 2.00 & 1.88 & 1.68 & 1.66 & $94.1 \pm 7$ & $84.1 \pm 4$ & $83.1 \pm 2$ \\
\hline \multirow[t]{3}{*}{ River water } & 0.00 & nd & nd & nd & & & \\
\hline & 0.50 & 0.47 & 0.49 & 0.48 & $94.7 \pm 5$ & $97.6 \pm 8$ & $96.7 \pm 7$ \\
\hline & 2.00 & 1.99 & 1.93 & 1.97 & $99.3 \pm 5$ & $96.6 \pm 2$ & $98.4 \pm 2$ \\
\hline \multirow[t]{3}{*}{ Wastewater } & 0.00 & nd & $0.11 \pm 5$ & nd & & & \\
\hline & 0.50 & 0.34 & 0.42 & 0.43 & $67.3 \pm 3$ & $83.9 \pm 6$ & $86.1 \pm 4$ \\
\hline & 2.00 & 1.18 & 1.37 & 1.62 & $59.0 \pm 4$ & $68.6 \pm 5$ & $80.9 \pm 4$ \\
\hline
\end{tabular}

a The DD-disk system was used

b Mean and relative standard deviations for determinations performed in triplicate

${ }^{c}$ Not detected 
The role of the supporting material (naked QFP) in the adsorption of the alkylphenols was also studied. Water samples $(1000 \mathrm{~mL})$ spiked with 0.50 or $2.00 \mathrm{ng} \mathrm{mL}^{-1}$ of the model analytes were enriched by applying single-layered naked QFP, double-layered naked QFP or triple-layered naked QFP. At the level of $0.50 \mathrm{ng} \mathrm{mL}{ }^{-1}$, none of the analytes were adsorbed onto the filters. However, at the level of $2.00 \mathrm{ng} \mathrm{mL}^{-1}$, about $3-4 \%$ and $3-11 \%$ of alkylphenols were retained by the double-layered and triple-layered naked QFPs, respectively. The adsorption ability of the naked QFPs was assessed still further, and $100-1000 \mathrm{~mL}$ volumes of water samples spiked with $10 \mathrm{ng}$ $\mathrm{mL}^{-1}$ of alkylphenols were concentrated by the triplelayered naked QFP. The recoveries of the components decreased drastically with increasing sample volume. When the volume was increased to $1000 \mathrm{~mL}$, the recoveries of BPA, 4-OP and 4-NP decreased to 0.6, 5.0 and $10 \%$ respectively, which demonstrated that the QFP played a minor role in the SPE of the three analytes when the SWCNT disk was used. Therefore, the adsorption of the alkylphenols was basically caused by the SWCNT adsorbent.

\section{Effect of flow rate}

Since the fast sample loading flow rate is one of the advantages of using SPE disks, the effect of flow rate on the extraction of alkylphenols was investigated by pretreating $500 \mathrm{~mL}$ of solution spiked with $10 \mathrm{ng} \mathrm{mL}^{-1}$ of BPA, 4-NP and 4-OP. The results showed that the sample loading flow rate for one SWCNT disk could be as high as $200 \mathrm{~mL} \mathrm{~min}^{-1}$. However, the extraction ability of the disk decreased significantly when the flow rate was faster than

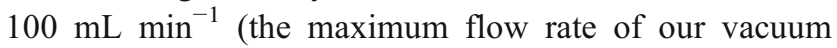
pump on the $\mathrm{C}_{18}$ disk). As the flow rate increased from 10 to $100 \mathrm{~mL} \mathrm{~min}{ }^{-1}$, the recoveries of BPA, 4-OP and 4-NP barely changed (see Fig. S1). For 4-NP, the recovery was still $>80 \%$ as the flow-rate reached $200 \mathrm{~mL} \mathrm{m^{-1 }}$. For the double- or triple-layered disks, the maximum flow-rate

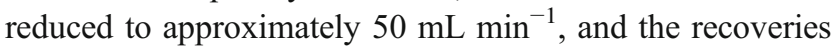
of all of the analytes remained constant as the sample loading rate was varied in the range of $10-50 \mathrm{~mL} \mathrm{~min}^{-1}$. Actually, the maximum flow rate of the triple-layered disk was comparable with that attained for the activated carbon disks with our vacuum pump. The DD-disk system was therefore used for real-world water samples, and the

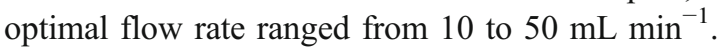

\section{Comparison study}

To further evaluate the extraction ability of the SWCNT disks, three groups of compounds with different polarities (including four phthalate esters, BPA, 4-NP, 4-OP, and five chlorophenols) were used as model compounds. A compar- ison study was conducted with a $\mathrm{C}_{18}$ disk and an activated carbon disk. Different volumes of aqueous solution spiked with $10 \mathrm{ng} \mathrm{mL} L^{-1}$ of each analyte were extracted by the SWCNT DD system, the $\mathrm{C}_{18}$ or the activated carbon disk.

Methanol was used as a powerful eluent for all the analytes extracted by the $\mathrm{C}_{18}$ and SWCNTs disks. Eight to fifteen milliliters of methanol were required to desorb all of the target compounds retained on the SWCNT disks, while $7 \mathrm{~mL}$ eluent was enough to desorb all of the analytes on the $\mathrm{C}_{18}$ disk. However, in the case of the activated carbon disk, methanol and acteonitrile did not enable the desorption of analytes. Quantitative recovery of phthalate esters was achieved with $80 \mathrm{~mL}$ of methylene chloride (Fig. 3C). For BPA, 4-NP, 4-OP and the chlorophenols, acetone was more efficient than methylene chloride and tetrahydrofuran. However, even with $150 \mathrm{~mL}$ of acetone, the recoveries of BPA, 4-NP and 4-OP were less than $60 \%$. Only $10 \%$ of the chlorophenol analytes was recovered with $100 \mathrm{~mL}$ of acetone. After extraction by activated carbon disk, the percolates of chlorophenols and alkylphenols (with different volumes) were collected and preconcentrated with the MWCNT cartridge $\left(250 \mathrm{mg}\right.$ ) or the $\mathrm{C}_{18}$ disk, respectively. As a result, none of the analytes was detected in the percolate. This indicated that the activated carbon disk had a high adsorption ability for these analytes, and that the low recoveries were caused by their irreversible interactions with the activated carbon adsorbent. This also means that applications of activated carbon disks are limited for the pretreatment of environmental water samples.

Comparisons between the SWCNT disks and the $\mathrm{C}_{18}$ disk are shown in Figs. 3, 4 and 5. It was observed that the DD-disk system possessed an exceptionally great ability,

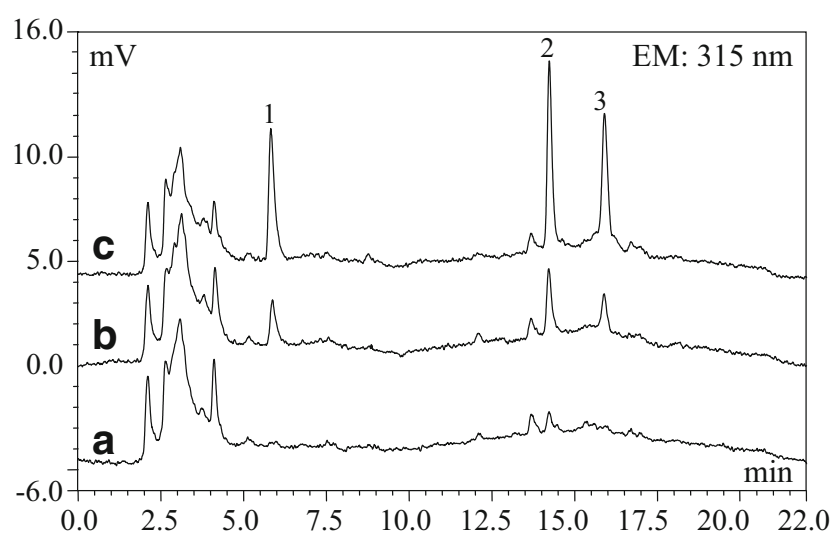

Fig. 6 LC-FLD chromatograms for the wastewater samples preconcentrated by SPE disks based on SWCNT sheets. A Wastewater sample; B wastewater sample spiked with $0.50 \mathrm{ng} \mathrm{mL}^{-1}$ of each compound; $\mathbf{C}$ wastewater sample spiked with $2.00 \mathrm{ng} \mathrm{mL}^{-1}$ of each compound. 1, Bisphenol A; 2, 4-tert-octylphenol; 3, 4-n-nonylphenol. Volume of water sample solution: $1000 \mathrm{~mL}$; volume of eluent (methanol): $10 \mathrm{~mL}$ 
just like the commercial $\mathrm{C}_{18}$ disk, to extract highly lipophilic compounds (the selected phthalate esters: $\log K_{\mathrm{ow}}$ 3.24-8.1; 4-OP: $\log K_{\text {ow }} 4.12$; and 4-NP: $\log K_{\text {ow }} 4.48$ ) from aqueous solutions. Also, the recoveries of these compounds were still $>90 \%$ when the sample volumes were increased to $2000 \mathrm{~mL}$. The commercial $\mathrm{C}_{18}$ disk appeared to be superior to the DD system for the preconcentration of a moderately lipophilic compound, BPA $\left(\log K_{\mathrm{ow}}, 3.18\right)$. For the chlorophenol analytes, however, the situation was reversed. The DD system was also more powerful than the $\mathrm{C}_{18}$ disk. For the moderately polar monochlorophenols (3-chlorophenol, $\log K_{\mathrm{ow}} 2.5$; and 4-chlorophenol, $\log K_{\mathrm{ow}}$ 2.4), the extraction efficiency of the DD system was 25 times higher than that of the $\mathrm{C}_{18}$ disk. Generally, two parameters, the specific surface area and the hydrophobicity of the reverse adsorbent, can affect the adsorption of organic analytes. Numerous research works have shown that the special crystalline structure of TNs (including the sidewall, which consists of vast hexagon-rich $\mathrm{sp}^{2}$ carbons and more reactive fullerene-like tips) can provide strong interactions with organic compounds (such as nonpolar, moderately polar and polar compounds). Additionally, compared with the results obtained in our previous work [8-10], the DD-disk SWCNT system (with $60 \mathrm{mg}$ of SWCNT adsorbent) showed the same high extraction ability for these selected analytes as 500 or $300 \mathrm{mg}$ of MWCNTs packed into a cartridge. Therefore, the highly hydrophobic nature of the SWCNTs and the greatly increased surface area of the disk both contribute to the high extraction ability of the SWCNT disk for the analytes in our study.

Method performance and sample analyses

The DD system was used to extract BPA, 4-OP and 4-NP from various $1000-\mathrm{mL}$ real-world water samples. Calibration curves were linear over the range $0.1-20 \mathrm{ng} \mathrm{mL}^{-1}$ with correlation coefficients of $>0.99$. The limits of detection for the extraction of the $1000-\mathrm{mL}$ water samples were calculated as three times the standard deviation of six replicate runs of procedure blanks. The results are shown in Table 1.

Several environmental water samples were analyzed using the DD system. None of the analytes were detected in tap water and river water, and only trace levels of 4-OP were found in wastewater (as shown in Table 2). The accuracy of the proposed method was evaluated from recovery studies performed by spiking water samples with 0.50 or $2.00 \mathrm{ng} \mathrm{mL}^{-1}$ of each analyte. The results are listed in Table 2. Satisfactory recoveries of all of the analytes were achieved from the tap water and river water samples. The relatively low recoveries of the analytes from the wastewater sample might be caused by the competitive adsorption of large amounts of dissolved organic carbon
(DOC) in wastewater. Chromatograms for the wastewater sample and its spiked solution are shown in Fig. 6.

\section{Conclusions}

A new, easy-to-make and practical solid-phase extraction disk based on a SWCNT sheet was investigated in this study for the first time. Satisfactory disk reproducibility was obtained for three analytes: BPA, 4-NP, and 4-OP. The perfect mechanical properties and the larger specific area of the disk permitted the use of higher flow rates. A comparison study showed that the DD-disk system (comprising two stacked SWCNT disks) with $60 \mathrm{mg}$ of SWCNT adsorbent exhibited extraction capabilities that were comparable to those of a commercial $\mathrm{C}_{18}$ disk with $500 \mathrm{mg}$ adsorbent for nonpolar or moderately polar compounds. The former system was more powerful than the latter for extracting polar analytes. Most importantly, unlike the situation for activated carbon disks, the analytes adsorbed on SWCNT disks were easily desorbed with $8-15 \mathrm{~mL}$ of methanol or acetonitrile. The DD-disk system was then used to preconcentrate several $1000-\mathrm{mL}$ environmental water samples spiked with a mixture of BPA, 4-NP and 4-OP, and satisfactory results were obtained. In summary, the new SPE disk proposed here has already shown some attractive features, such as strong extraction abilities for a wide range of compounds with different polarities, high extraction speed, and easy preparation; furthermore, it has shown great analytical potential for the SPE of large-volume real-world water samples.

Acknowledgements This work was jointly supported by the National Basic Research Program of China (2003CB415001); the National Natural Science Foundation of China (20475060, 20621703) and the Major Research Program of the Chinese Academy of Sciences (KZCX2-YW-420-1).

\section{References}

1. Fritz JS, Masso JJ (2001) J Chromatogr A 909:79-85

2. Thurman EM, Snavely K (2000) Trend Anal Chem 19:18-26

3. Westbom R, Thörneby L, Zorita S, Mathiasson L, Björklund E (2004) J Chromatogr A 1033:1-8

4. Ferrer I, Barceló D, Thurman EM (1999) Anal Chem 71:1009-1015

5. Leandro CC, Bishop DA, Fussell RJ, Smith FD, Keely BJ (2006) J Agric Food Chem 54:645

6. Slobodník J, Öztezkizan Ö, Lingeman H, Brinkman UATh (1996) J Chromatogr A 750:227-238

7. Hennion MC (2000) J Chromatogr A 885:73-95

8. Cai YQ, Jiang GB, Liu JF, Zhou QX (2003) Anal Chem 75:25172521

9. Cai YQ, Jiang GB, Liu JF, Zhou QX (2003) Anal Chim Acta 494:149-156

10. Cai YQ, Cai YE, Mou SF, Lu YQ (2005) J Chromatogr A 1081:245-247 
11. Zhou QX, Xiao JP, Wang WD, Liu GG, Shi QZ, Wang JH (2006) Talanta 28:1309-1315

12. Biesaga M, Pyrzynska K (2006) J Sep Sci 29:2241-2244

13. Niu HY, Cai YQ, Shi YL, Wei FS, Liu JM, Mou SF, Jiang GB (2007) Anal Chim Acta 594:81-92

14. Baughman RH, Cui C, Zakhidov AA, Iqbal Z, Barisci JN, Spinks GM, Wallace GG, Mazzoldi A, de Rossi D, Rinzler AG, Jaschinski O, Roth S, Kertesz M (1999) Science 284:1340-1344

15. Landi BJ, Raffaelle RP, Heben MJ, Alleman JL, VanDerveer W, Gennett T (2002) Nano Lett 2:1329-1332

16. Spinks GM, Wallace GG, Fifield LS, Dalton LR, Mazzoldi A, Rossi DD, Khayrullin II, Baughman RH (2002) Adv Mater 14:1728-1732

17. Penza M, Cassano G, Aversa P, Antolini F, Cusano A, Giordano M, Nicolais L (2004) Appl Phys Lett 85:2379-2381
18. Whitten GP, Spinks GM, Wallace GG (2005) Carbon 43:1891-1896

19. Shiraishi S, Kurihara H, Okabe K, Hulicova D, Oya A (2002) Electrochem Commum 4:593-598

20. Vohrer U, Kolaric I, Haque MH, Roth S, Detlaff-Weglikowska U (2004) Carbon 42:1159-1164

21. Novak JP, Snow ES, Houser EJ, Park D, Stepnowski JL, McGill RA (2003) Appl Phys Lett 83:4026-4028

22. Zheng F, Baldwin DL, Fifield LS, Anheier NC, Aardahl CL, Grate JW (2006) Anal Chem 78:2442-2446

23. Berhan L, Yi YB, Sastry AM, Munoz E, Selvidge M, Baughman RH (2004) J Appl Phys 95:4335-4345

24. Vodenitcharova T, Mylvaganam K, Zhang LC (2007) J Mater Sci 42:4935-4941

25. Cinke M, Li J, Chen B, Cassell A, Delzeit L, Han J, Meyyappan M (2002) Chem Phys Lett 365:69-74 